\title{
NLRs, BLRs and Starbursts at high redshift
}

\author{
Hagai Netzer ${ }^{1}$ \\ ${ }^{1}$ School of Physics and astronomy, Tel Aviv University, Tel Aviv 69978, Israel
}

\begin{abstract}
New infrared spectroscopy of 22 high-luminosity high-redshift AGNs helps to test several suggestions regarding the mass, composition and size of the emission line regions in active galacic nuclei (AGN). A plot of the metalicity luminosity correlation over more than five orders of magnitude in luminosity shows that most narrow line Seyfert 1 galaxies fall outside of the general trend. However, when $L / L_{E d d}$ is plotted instead of luminosity, all sources follow the same trend suggesting this to be the primary driver of the correlation. Regarding the narrow emission lines in the most luminous sources, there is a dichotomy in the observed properties where $2 / 3$ of the sources show very strong [O III] $\lambda 5007$ lines while the remaining $1 / 3$ have no detectable NLR. On the basis of this I argue that earlier proposed relations of the type $R_{N L R} \propto L_{\text {[OIII] }}^{1 / 2}$ must break down for $R_{N L R}$ exceeding a few kpc. Thus, NLRs in high luminosity AGN may be very different from those observed in nearby sources. In particular, some high luminosity sources may be in a phase of violent star-formation that produce a large quantity of high density gas in their central kpc.
\end{abstract}

\section{Scaling and weighing the BLR}

Decade long studies of the broad line region (BLR) in active galactic nuclei (AGN) have shown that the BLR size (i.e. the emissivity weighted radius of the $\mathrm{H} \beta$ emitting region) is

$$
R_{B L R} \simeq 0.4\left[\frac{\lambda L_{\lambda}(5100 \AA)}{10^{46} \mathrm{erg} \mathrm{s}^{-1}}\right]^{0.6} \mathrm{pc}
$$

(e.g. Kaspi et al 2000). This simple scaling law allows a rough estimate of the total mass of the gas emitting the broad emission lines which is

$$
M_{B L R} \simeq 1.8 \times 10^{2}\left[\frac{\lambda L_{\lambda}(5100 \AA)}{10^{46} \mathrm{erg} \mathrm{s}^{-1}}\right]^{1.2}\left[\frac{C_{f}}{0.1}\right]\left[\frac{N_{H}}{10^{23} \mathrm{~cm}^{-2}}\right] M_{\odot} .
$$

Here $C_{f}$ is the $(4 \pi)$ covering factor and $N_{H}$ is the hydrogen column density of a "typical" cloud which is assumed to be an order of magnitude larger than the column density of the highly ionized part of the cloud. Thus, for the most luminous quasars $\left(\lambda L_{\lambda}(5100 \AA)=\right.$ few $\times 10^{47} \mathrm{erg} \mathrm{s}^{-1}$ ) the mass associated with this region is of order $10^{4} M_{\odot}$. The origin of this large amount of gas is basically unknown. It is therefore important to understand some of its other properties, in particular its chemical composition.

\section{Metalicity, accretion rate and star-formation in AGN}

Early abundance studies in AGN utilized weak and broad intercombination lines, such as $\mathrm{N}$ III] $\lambda 1750, \mathrm{~N}$ IV] $\lambda 1486$, and OIII] $\lambda 1663$, to determine metal abundances in the BLR gas (Shields 1976). Hamann \& Ferland (1993; hereafter HF93) found that emission-line ratios, such as Nv $\lambda 1240 / \mathrm{CIV} \lambda 1549$ (hereafter Nv/CIV) can trace the BLR metalicity 
across a wide range of luminosity and BLR density. HF93 also found that BLR metalicity, determined in this way, correlates with luminosity, in what they proposed as the metalicity-luminosity $(Z-L)$ relationship. Given the well known relationship between AGN luminosity and black hole mass $\left(M_{\mathrm{BH}}\right)$, HF93 suggested that the $Z-L$ relationship can naturally lead to a $Z-M_{\mathrm{BH}}$ dependence, in analogy with the mass-metalicity relation observed in some elliptical galaxies.

Shemmer \& Netzer (2002; hereafter SN02) have shown that once narrow-line Seyfert 1 galaxies (NLS1s) are introduced to the $Z-L$ diagram, they deviate significantly from the $Z-L$ relation by exhibiting high $\mathrm{Nv} / \mathrm{CIV}$ at low luminosity. A possible explanation is that Nv/Civ is not an adequate metalicity indicator for NLS1s (and perhaps other AGNs). According to SN02, BLR metalicity also depends on the width of $\mathrm{H} \beta$, which is perhaps the best accretion rate (in terms of the Eddington ratio, $L / L_{E d d}$ ) indicator, and that this dependence may prevail also at the high-luminosity end.

In a recent paper (Shemmer et al. 2004, hereafter S04) we have tested this idea by computing the mass and accretion rate in a sample of 22 high redshift high luminosity AGN. All of our quasars lie in a $2<z<2.6$ redshift range, with $\mathrm{H} \beta$ well inside the $H$ band. Observations were done at the Anglo-Australian Telescope (AAT) in Australia and at Telescopio Nazionale Galileo (TNG) in Spain. The $\mathrm{H} \beta$ line width was measured in all sources and was used, together with the $5100 \AA$ continuum luminosity, to obtain $M_{B H}$ and $L / L_{E d d}$. We supplemented those observations with UV and optical data to obtain a sample of 85 AGN covering more than five orders of magnitude in luminosity. All object have measurable $\mathrm{H} \beta$ and $\mathrm{Nv} / \mathrm{CIV}$.

The main results of this study are:

(a) The Nv/Civ line ratio correlates poorly with both $L$ and $M_{B H}$ mostly because of the presence of a group of NLS1s with large Nv/CIV yet very low luminosity.

(b) The Nv/Civ correlates strongly with $L / L_{E d d}$ suggesting that the latter is the main driver for the earlier noted metalicity-luminosity relationship. This correlation is shown in Fig. 1. Part of the scatter in the $Z-L / L_{\mathrm{Edd}}$ diagram may be contributed by the yet unknown effect of BLR density on the way BLR metalicity is derived. The problem has been mentioned by HF93 but the observational verification is still missing.

(c) Using CIV $\lambda 1549$ as a proxy to $\mathrm{H} \beta$ in estimating $M_{B H}$ might be problematic and lead to spurious mass and accretion rate estimates in individual sources.

The result of this study may hint for the origin of the BLR mass as well as to the high metalicity of this component. S04 speculated that violent star formation events may be the origin of the nuclear gas. The supply of this enriched material might have triggered a phase of large accretion rate which in tern resulted in unusual luminosity. The highest luminosity quasars are in the midst of such a phase. The nearby low luminosity NLS1s are in a similar phase associated with a much smaller black hole. The new results are consistent with a scenario in which there is an intimate relation between AGN fueling, manifested by accretion rate, and starburst, responsible for the metal-enrichment.

\section{Scaling and weighing the NLR}

The narrow line regions (NLRs) have been studied, extensively, from the ground and from space. This component of the nucleus is spatially resolved up to a redshift of about 0.5. Thus, detailed NLR mappings are now available for a large number of sources covering a large range of luminosity and redshift (Falcke, Wilson, \& Simpson 1998; Bennert et al. 2002, hereafter B02, Schmitt et al. 2003).

Several recent NLR studies lead to apparently conflicting results. BO2 obtained narrow band HST images of seven luminous radio-quiet Palomar-Green quasars with $z<0.5$. 


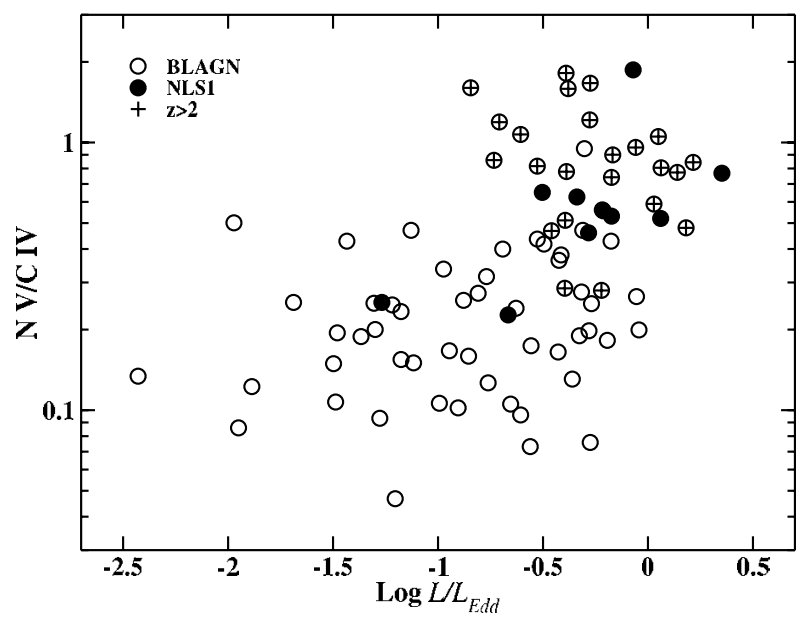

Figure 1. Nv/CIV vs. accretion rate for the S04 sample. Empty circles are AGNs with $\mathrm{FWHM}(\mathrm{H} \beta)>1500 \mathrm{~km} \mathrm{~s}^{-1}$, and empty circles with plus signs indicate $z>2$ quasars. NLS1s are marked by filled circles. A strong metalicity-accretion rate correlation for all AGNs is apparent, and most NLS1s are found in the same region of parameter space that is shared by the high- $z$ quasars.

They suggest that the NLR size (radius) scales with the [O III] $\lambda 5007$ and the $\mathrm{H} \beta$ line luminosities and is given by

$$
R_{N L R} \simeq 2.1 L_{[O I I I], 42}^{0.52 \pm 0.06} \mathrm{kpc},
$$

where $L_{[O I I I], 42}=L_{[O I I I]} / 10^{42} \mathrm{erg} \mathrm{s}^{-1}\left(\mathrm{H}_{0}=70 \mathrm{~km} \mathrm{~s}^{-1} \mathrm{Mpc}^{-1}, \Omega_{m}=0.3\right.$, and $\left.\Omega_{\Lambda}=0.7\right)$. The above $R_{N L R}$ can be translated to the mass of the NLR gas which is approximately

$$
M_{N L R} \simeq 10^{9}\left[\frac{C_{f}}{0.1}\right]\left[\frac{N_{H}}{10^{21} \mathrm{~cm}^{-2}}\right]\left[\frac{R_{N L R}}{10 \mathrm{kpc}}\right]^{2} M_{\odot} .
$$

According to this expression, the most luminous sources contain very much gas in their huge NLRs. This dependence has been questioned by Schmitt et al. (2003) who studied a much larger sample albeit with much lower luminosity, and found $R_{N L R} \propto L^{0.33}$. A new work by Bennert et al. (2004, these proceedings) argues that much of the difference is due to orientation since most of the sources in Schmitt et al. (2003) are Seyfert 2s while the more luminous sources in B02 are all type-I AGNs.

Croom et al. (2002) analyzed the spectra of $\sim 22,000$ AGNs from the 2dF quasar redshift survey (2QZ) and claimed to see a decrease in the equivalent width (EW) of several narrow lines $([\mathrm{OII}] \lambda 3727,[\mathrm{NeV}] \lambda 3426$ and $[N e I I I] \lambda 3870)$ with source luminosity. They suggested that at least part of this "Baldwin effect" (Baldwin 1977) is due to the increase in NLR size with source luminosity which leads to galactic-scale dimensions in the most luminous objects. Such NLRs are likely to escape the system leading to AGNs with weak or no NLR emission.

A real physical explanation of any $R-L$ dependence is still lacking. Some papers assume a stratified, radiation bounded NLR with a pre-chosen run of density and hence level of ionization (e.g., Netzer 1990; Komossa \& Schulz 1997). Such models are naturally normalized in incident flux units $\left(L_{i o n} / R^{2}\right)$ and can be tuned to produce the same mean ionization parameter for all sources (see however density bounded models e.g. Binette, Wilson, \& Storchi-Bergman 1996). An almost orthogonal approach is provided by the "locally optimally emitting cloud" (LOC) model (Ferguson et al. 1997). This model 


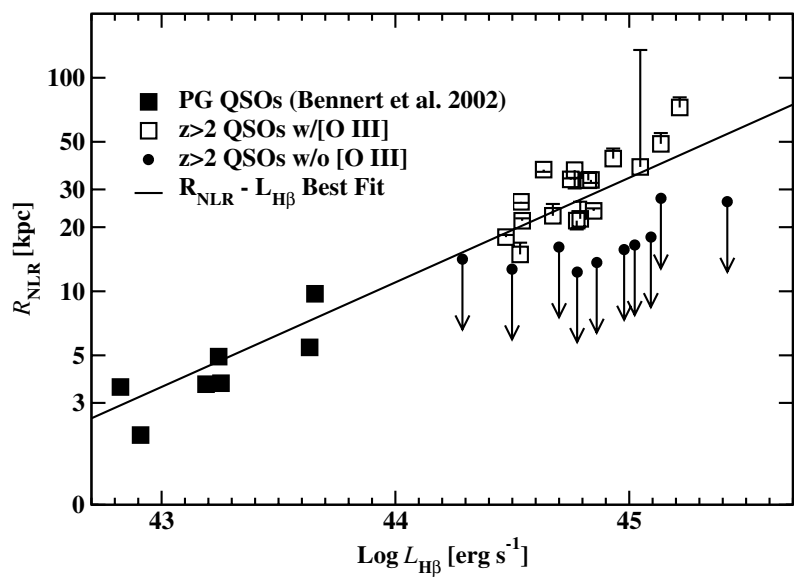

Figure 2. $R_{N L R}$ vs. $L$ for luminous quasars. Filled squares are the original B02 quasar data and the straight line is the $\mathrm{B} 02 R_{N L R}-L_{\mathrm{H} \beta}$ best-fit line. Open symbols represent 18 high- $z$ quasars with measured [O III] $\lambda 5007$ lines, for which $R_{N L R}$ was inferred from the $\mathrm{B} 02 R_{N L R}-L_{[\mathrm{OIII}]}$ relation. Arrows pointing down represent 10 high- $z$ quasars with upper limits only. Note the two distinct groups and the enormous predicted $R_{N L R}$ at large $\mathrm{H} \beta$ luminosity (from N04).

provides a natural scaling of $L_{i o n}$ with $R_{N L R}$ provided there is a large reservoir of gas with similar properties in all AGNs and on all scales. The recent papers by Dopita et al. (2002) and Gvoves et al. (2004, see also these proceedings) provide a more solid foundation to NLR modeling. These authors assumed dusty, stratified NLR clouds where the external radiation pressure acts mostly on the dust particles and forces the local ionization parameter to certain specific values. The model explains naturally the $R_{N L R} \propto$ $L^{1 / 2}$ dependence.

\section{Disappearing NLRs}

The combination of the B02 observations and the theoretical arguments for a "natural" $R_{N L R} \propto L_{i o n}^{1 / 2}$ dependence raises a severe problem regarding the NLR size in high luminosity AGN. This has recently been discussed in Netzer et al. (2004, hereafter N04) which is based on the same data set shown in S04. Fig. 2 which is taken from N04 shows three quantities vs. $L_{H \beta}\left(\right.$ broad). The first is the measured $R_{N L R}$ from the B02 sample (seven sources). The second is from our newly observed $\mathrm{H} \beta$ and [O III] $\lambda 5007$ lines with two additional sources from Dietrich et al. (2002). For these we use Eq. 3.1 to guess $R_{N L R}$, given the observed $L_{[O I I I]}$. The third group includes six sources from the S04 sample and four sources from Dietrich et al. (2002) where no [O III] $\lambda 5007$ has been detected. For these we use $R_{N L R}$ derived from the upper limits on $L_{[O I I I]}$. The upper limits on $R_{N L R}$ obtained in this way are a factor of $2-3$ smaller than those derived from $L_{H \beta}$.

The implications of Fig. 2 are clear. For those sources with measured $\mathrm{H} \beta$ and [O III] $\lambda 5007$ lines, the derived $R_{N L R}$ is enormous, exceeding, in the most luminous sources, $100 \mathrm{kpc}$ in diameter. I consider those sizes completely unreasonable for reasons that are discussed in detail in N04. The diagram also suggests a dichotomy in the properties of the high luminosity quasars, where some sources show strong [O III $] \lambda 5007$ lines and other show no or very weak such emission.

We are facing a situation where sound physical arguments support the expected $R_{N L R} \propto$ $L^{1 / 2}$ relationship yet its application to the most luminous quasars give unreasonably large sizes. A possible solution is that "typical" NLRs (i.e. those similar in their properties to 
the ones observed in Seyfert 1 galaxies) cannot last very long in high and perhaps also in intermediate luminosity AGNs. This means that the [O III $] \lambda 5007$ emitting regions in the highest luminosity sources may be of a different nature and different physical properties, in particular they are much smaller than inferred from the simple $R-L$ scaling relationships.

\section{Luminous NLRs as star forming regions}

If compact NLRs are indeed typical of many high luminosity AGNs, then their properties must be very different from the properties of those NLRs observed in lower luminosity sources. In particular, the gas density in the high luminosity NLRs must be several orders of magnitude larger than the typical densities in the lower luminosity sources. The spectroscopic properties must be very different too which can, in principle, be tested by accurate observations.

A possible origin of a high density gas in kpc-scale nuclear regions is intense starforming activity. Such events can be associated with high density, large column density, non-solar composition dusty gas. The overall spectrum of such regions is likely to differ from the spectrum of nearby, lower density NLRs.

A possible scenario that explains the observations of high luminosity AGNs, and the apparent break down of the $R_{N L R} \propto L_{i o n}^{1 / 2}$ relationship, is of two distinct populations. One where such scaling continues to high luminosity due to radiation pressure force or other effects. This results in enormous NLRs that leave the system on short time scales. Such objects will have basically no nuclear narrow emission lines. The other group is those sources where starburst or another unknown process ejects high density gas into their nuclear region. This gas is ionized and excited by the central radiation source and produces the observed strong [O III] $\lambda 5007$ lines. Such "star-forming NLRs" would have spectral properties that are different from those observed in near-by less luminous sources.

\section{Acknowledgements}

I am grateful to the technical staff at the AAT and TNG observatories for invaluable help in the observations. This review is based on two recently submitted papers, S04 and N04. I am most grateful to all my co-authors on those papers: O. Shemmer, R. Maiolino, E. Oliva, S. Croom, E. Corbett and L. di-Fabrizio. This work is supported by the Israel Science Foundation grant 232/03.

\section{References}

Baldwin, J. A., 1977 ApJ, 214, 679

Binette, L., Wilson, A. S., \& Storchi-Bergmann, T. 1996, A\&A, 312, 365

Bennert, N., Falcke, H., Schulz, H., Wilson, A. S., \& Wills, B. J. 2002, ApJ, 574, L105 (B02)

Bennert, N., Falcke, H., Shchekinov, Y., \& Wilson, A. S. 2004, (2004, these proceedings)

Croom, S. M., et al. 2002, MNRAS, 337, 275

Dietrich, M., Appenzeller, I., Vestergaard, M., \& Wagner, S. J. 2002, ApJ, 564, 581

Dopita, M. A., Groves, B. A., Sutherland, R. S., Binette, L., \& Cecil, G. 2002, ApJ, 572, 753

Falcke, H., Wilson, A. S., \& Simpson, C. 1998, ApJ, 502, 199

Hamann, F. \& Ferland, G. 1993, ApJ, 418, 11 (HF93)

Hamann, F., Korista, K. T., Ferland, G. J., Warner, C., \& Baldwin, J. 2002, ApJ, 564, 592

Groves, B. et al. 2004 (ApJ in press; see also these proceedings)

Kaspi, S., Smith, P. S., Netzer, H., Maoz, D., Jannuzi, B. T., \& Giveon, U. 2000, ApJ, 533, 631

Komossa, S., \& Schulz, H. 1997, A\&A, 323, 31 
Netzer, H. 1990, in Active Galactic Nuclei, (eds., T. J.-L. Courvoisier \& M. Mayor), Berlin: Springer, 57

Netzer, H., Shemmer, O., Maiolino, R., Croom, S., Oliva, T., \& Di-Fabrizio, L. 2004, ApJ submitted (N04)

Schmitt, H. R., Donley, J. L., Antonucci, R. R. J., Hutchings, J. B., Kinney, A. L., \& Pringle, J. E. 2003, ApJ, 597, 768

Shemmer, O., \& Netzer, H. 2002, ApJ, 567, L19 (S02)

Shemmer, O., Netzer, H., Maiolino, R., Croom, S., Oliva, T., \& Di Fabrizio, L. 2004, ApJ, submitted (S04)

Shields, G. A. 1976, ApJ, 204, 330 